\title{
Nonlinear optical model for strip plasmonic waveguides
}

\author{
Lysenko, Oleg; Bache, Morten; Lavrinenko, Andrei
}

Published in:

Journal of the Optical Society of America B-optical Physics

Link to article, DOI:

10.1364/JOSAB.33.001341

Publication date:

2016

Document Version

Peer reviewed version

Link back to DTU Orbit

Citation (APA):

Lysenko, O., Bache, M., \& Lavrinenko, A. (2016). Nonlinear optical model for strip plasmonic waveguides. Journal of the Optical Society of America B-optical Physics, 33(7), 1341-1348.

https://doi.org/10.1364/JOSABB.33.001341

\section{General rights}

Copyright and moral rights for the publications made accessible in the public portal are retained by the authors and/or other copyright owners and it is a condition of accessing publications that users recognise and abide by the legal requirements associated with these rights.

- Users may download and print one copy of any publication from the public portal for the purpose of private study or research.

- You may not further distribute the material or use it for any profit-making activity or commercial gain

- You may freely distribute the URL identifying the publication in the public portal

If you believe that this document breaches copyright please contact us providing details, and we will remove access to the work immediately and investigate your claim. 


\title{
Nonlinear optical model for plasmonic waveguides
}

\author{
Oleg lysenko, Morten Bache, Andrei lavrinenko \\ Department of Photonics Engineering, Technical University of Denmark, Oersteds Plads, Building 345V, Kongens Lyngby 2800, Denmark \\ *Corresponding author: ollyse@fotonik.dtu.dk
}

Received XX Month XXXX; revised XX Month, XXXX; accepted XX Month XXXX; posted XX Month XXXX (Doc. ID XXXXX); published XX Month XXXX

\begin{abstract}
This paper presents a theoretical model of nonlinear optical properties for plasmonic waveguides. The particular waveguides geometry that we investigate contains a gold core, adhesion layers and silicon dioxide cladding. It is shown that the third-order susceptibility of the gold core significantly depends on the layer thickness and has the dominant contribution to the effective third-order susceptibility of the long-range plasmon polariton mode. This results in two nonlinear optical effects in plasmonic waveguides, which we experimentally observed and reported in 0pt. Lett. 41, 317 (2016). The first effect is the nonlinear power saturation of the plasmonic mode, and the second effect is the spectral broadening of the plasmonic mode. Both nonlinear plasmonic effects can be used for practical applications and their appropriate model will be important for further developments in communication approaches. (C) 2015 Optical Society of America
\end{abstract}

OCIS codes: (250.5403) Plasmonics; (230.7370) Waveguides; (190.4400) Nonlinear optics, materials; (240.6680) Surface plasmons.

http://dx.doi.org/10.1364/AO.99.099999

\section{INTRODUCTION}

The optically controlled optical channel is considered as one of the main tools in enhancing density of communication lines with reduced energy-per-bit consumption. All-optical signal processing is capable of achieving a signal modulation and transmission speeds beyond what is possible by their electronic counterparts. To optically modulate (or switch) signals in plasmonic waveguides (with one or several metaldielectric interfaces) means that light induces changes in the real or imaginary parts of the dielectric functions of the constituent materials. These changes can occur either in a metal or in a dielectric layer, or in both. While dielectric nonlinearities are well-known, metal nonlinearities are not deeply explored. Most of the studies in nonlinear plasmonics are connected with the local field enhancement facilitated by metal nanoparticles [1].

So far the light-induced absorption modulation has been studied with the intrinsic metal nonlinearity [1]. The intrinsic nonlinearity of gold is related to the interband excitation of non-equilibrium electrons and results in small changes of the real part of the permittivity but in significant changes of the imaginary part. The associated relaxation time is on the picosecond scale, making THz-frequency modulation of signals in plasmonic gold waveguides possible. The relaxation time of the interband transitions in aluminum is much smaller and can provide even faster modulation speeds [2]. Thus, the optical nonlinearity in metals holds a potential that needs to be explored more. Results can be exploited for rapid optical switching and modulation by using strong excitation via an ultrafast laser pulse. This approach has opened a whole new field of active plasmonic systems [2].

Recent experiments confirm the presence of nonlinear propagation effects in plasmonic waveguides, much like the ones that for decades have been fueling nonlinear fiber optics [3]. We have conducted recently an experiment [4] with strip plasmonic gold waveguides and observed that the loss performance was nonlinear as the power was increased. We attribute this effect to the imaginary part of the nonlinearity of gold. We also saw indications of self-phase modulation in picosecond pulses spectra governed by the real part of the thirdorder susceptibility. Both effects were significantly affected by the nanometer-scale thickness of the gold layers as one can conclude from the restored effective nonlinearity parameters. To our knowledge such a study has not yet been done in plasmonic waveguides. An early study [5] used the cw-like pumping and obtained significant thermal contributions to the nonlinear phase. Also, the real and imaginary components of the third-order susceptibility of thin gold layers were characterized using the z-scan method [6, 7]. In another recent experiment Baron et al. [8] measured the imaginary component of the third-order susceptibility for bulk gold by studying nonlinear propagation of surface-plasmon polaritons at a single air/metal interface.

The present paper provides the theoretical background to our recent experiment, as to understand and ultimately exploit intrinsic metal nonlinearities in thin metal films. The posed problem is tricky, because the nonlinear metal properties are not yet well understood. The current vision of possible nonlinear behavior in noble metals is that they occur due to the Fermi smearing process. When a thin metal film is exposed to an ultrafast laser pulse, it absorbs the optical pulse which leads to rapid heating. The rise in the temperature of the material results in interband transitions and broadens the electronic distribution around the Fermi energy. This eventually causes significant change in the dielectric permittivity of metal [9]. Another possible mechanism is that a strong electric field in ultra-short laser pulses affects the distribution of free electrons. Such direct influence is preferable in terms of exploitation due to the fast electronic response and symmetric time scale of both phases of the nonlinear process: development and relaxation. 
In our recent experiment we characterized strip plasmonic waveguides, and this geometry forms the basis of the theory in the present paper. The waveguide layout contains the gold core with the finite microscale width and nanoscale thickness, tantalum pentoxide adhesion layer and silicon dioxide cladding. This particular waveguide configuration was chosen because it provides effective propagation of a long-range surface plasmon polariton (LRSPP) [10]. The optical properties of the LRSPP mode are utilized in many applications, such as nanofocusing of electromagnetic radiation [11] or photodetectors [12]. The nonlinear effects associated with propagation of a LRSPP in plasmonic waveguides are less investigated. One theoretical approach is related to application of the hydrodynamic model for the freeelectron gas [13]. In other works, the nonlinear propagation of LRSPP modes is studied in terms of either the cascaded second-order nonlinearity at a metal-dielectric interface [14] or the third-order nonlinearity of gold and silicon dioxide in plasmonic nanorod structures [15]. In the latter case, the third-order nonlinearity for gold was obtained from the two-temperature model of electrons dynamics [16] and the ideal lossless metal was assumed [15]. In the present paper we take into account that all constituent materials (metal, adhesion layer, and cladding) exhibit a nonlinear response. So, in line with the classical nonlinear fiber optics formalism we introduce an effective nonlinearity of the LRSPP waveguide mode. Additionally we also consider losses originated both from the linear and nonlinear parts of the metal dielectric function. The effective nonlinearity of the LRSPP mode leads to two interesting nonlinear effects. The first effect is a nonlinear saturation of the average power of the LRSPP mode. The second effect is nonlinear phase modulation with the spectral broadening of the LRSPP pulse. Since our experiment was conducted with a powerful picosecond laser at $1064 \mathrm{~nm}$, we apply our model for this specified wavelength. We also discuss other important ultrafast laser wavelengths $800 \mathrm{~nm}$ and $1550 \mathrm{~nm}$, in particular, regarding different gold behavior.

The article has the following structure. Section 2 describes optical properties of the constituent materials: gold, tantalum pentoxide and silicon dioxide. Section 3 presents the effective index theory for calculation of the LRSPP propagation constant in the strip plasmonic waveguide. Section 4 explains a role of the chromatic dispersion in propagation of the LRSPP mode. Section 5 is devoted to nonlinear parameters of the plasmonic waveguide. Section 6 shows a solution to the pulse propagation equation for the LRSPP mode and explains the obtained nonlinear effects.

\section{OPTICAL PROPERTIES OF MATERIALS}

We will start the modeling of plasmonic waveguides by considering optical properties of the constituent materials. Dielectric permittivity $\varepsilon_{\mathrm{SiO}_{2}}$ of silicon dioxide (silica) is described by the following Sellmeier equation [17]:

$\varepsilon_{\mathrm{SiO}_{2}}=1+\frac{0.6961663 \lambda^{2}}{\lambda^{2}-(0.0684043)^{2}}+\frac{0.4079426 \lambda^{2}}{\lambda^{2}-(0.1162414)^{2}}+\frac{0.8974794 \lambda^{2}}{\lambda^{2}-(9.896161)^{2}}$,

where $\lambda$ is the light wavelength in $\mu \mathrm{m}$. Permittivity $\mathcal{E}_{\mathrm{TPO}}$ of tantalum pentoxide (amorphous dielectric) is given by the following equation [18]:

$$
\varepsilon_{\mathrm{TPO}}=\varepsilon_{\infty}-\frac{\omega_{\mathrm{p}, 0}^{2}}{\omega^{2}+i \gamma_{0} \omega}+\sum_{j=1}^{6} \frac{\omega_{\mathrm{p}, \mathrm{j}}^{2}}{\omega_{j}^{2}-\omega^{2}-i \gamma \omega} .
$$

Here $\omega=2 \pi c / \lambda$ is the light angular frequency, $c$ is the vacuum speed of light, $\boldsymbol{\varepsilon}_{\infty}=\left(A+B / \lambda^{2}\right)^{2}$ is the dielectric permittivity of amorphous $\mathrm{Ta}_{2} \mathrm{O}_{5}$ from the Cauchy dispersion formula [18] with $A=$ 2.06 and $B=0.025 \mu \mathrm{m}^{2} ; \omega_{\mathrm{p}, 0}=6490 \mathrm{~cm}^{-1}$ and $\gamma_{0}=6.5 \times 10^{-5} \mathrm{~cm}^{-1}$ are the Drude plasma frequency and scattering rate for $\mathrm{Ta}_{2} \mathrm{O}_{5}$, and $\omega_{\mathrm{p}, \mathrm{j}}, \gamma_{\mathrm{j}}(j=1,2,3,4,5,6)$ are the experimental parameters for the Lorentz oscillators taken from Ref. [18]. The linear refractive indices are $n_{\mathrm{SiO}_{2}}=\sqrt{\boldsymbol{\varepsilon}_{\mathrm{SiO}_{2}}}$ for silica, and $n_{\mathrm{TPO}} \approx \sqrt{\mathrm{Re}\left[\boldsymbol{\varepsilon}_{\mathrm{TPO}}\right]}$ for tantalum pentoxide, respectively [Fig. 1(a)]. They have two main features. First, tantalum pentoxide has larger values of the linear refractive index: in particular at $1064 \mathrm{~nm}$, the refractive index $n_{\mathrm{SiO}_{2}}$ for silica is $\sim 1.45$, and the refractive index $n_{\text {TPO }}$ for tantalum pentoxide is $\sim 2.07$. Second, silica has a zero dispersion wavelength (ZDW) at $\sim 1.27 \mu \mathrm{m}$ [3], and tantalum pentoxide at $\sim 1.8 \mu \mathrm{m}$ [18].

Dielectric permittivity $\varepsilon_{\mathrm{Au}}$ of a thin gold layer is based on the classical Drude model [19], but with accounting for an electron confinement factor. For a metal nanosphere the frequency of electrons collisions $\gamma_{\mathrm{f}}$ depends on the radius $r[20,21]$. In the same way of arguing as in [20], where $1 / r$ dependence reflects the ratio of surface area to volume, we can proceed by assuming that for a thin gold stripe with thickness $t$ we can take:

$$
\begin{gathered}
\operatorname{Re}\left[\varepsilon_{\mathrm{Au}}\right]=1-\frac{\omega_{\mathrm{p}}{ }^{2}}{\gamma_{\mathrm{f}}{ }^{2}+\omega^{2}}, \\
\operatorname{Im}\left[\varepsilon_{\mathrm{Au}}\right]=\frac{\gamma_{\mathrm{f}} \omega_{\mathrm{p}}^{2}}{\omega\left(\gamma_{\mathrm{f}}^{2}+\omega^{2}\right)}, \\
\gamma_{\mathrm{f}}=\gamma_{\infty}+\frac{v_{\mathrm{F}}}{t},
\end{gathered}
$$

where $\omega_{\mathrm{p}}=\sqrt{n e^{2} / \varepsilon_{0} m_{\mathrm{e}}}$ is the plasma frequency for gold, $e$ is the electron charge, $\varepsilon_{0}$ is the vacuum permittivity, $n$ is the electron density, $m_{\mathrm{e}}$ is the electron mass, $\gamma_{\infty}=3.3 \times 10^{13} \mathrm{~s}^{-1}$ is the frequency of electrons collisions for bulk gold, and $v_{\mathrm{F}}$ is the Fermi velocity of electrons. As the layer thickness $t$ approaches nanoscale values, free electrons start to feel the layer boundaries and the second term in Eq. (5) accounts for this increment of the electrons collisions frequency, while the plasma frequency stays the same [19]. The local Drude model may not be applicable for layers with the thicknesses below 10 $\mathrm{nm}[13,22]$. Also there is an intensive clustering process for thin layers [20], and their fabrication especially with thicknesses below $10 \mathrm{~nm}$ is still challenging [23]. Therefore our model will focus on plasmonic waveguides with the thickness values above the clustering threshold. Constraining parameter $t$ by $10 \mathrm{~nm}$ it is straightforward to show that Eq. (4) can be simplified:

$$
\operatorname{Im}\left[\varepsilon_{\mathrm{Au}}\right] \approx\left(\operatorname{Im}\left[\varepsilon_{\mathrm{Au}}\right]\right)_{\infty}+\frac{\omega_{\mathrm{p}}^{2} v_{\mathrm{F}}}{\omega\left(\gamma_{\infty}^{2}+\omega^{2}\right) t},
$$

where $\left(\operatorname{Im}\left[\varepsilon_{\mathrm{Au}}\right]\right)_{\infty}$ is the imaginary part of the permittivity for bulk gold. 
The real and imaginary parts of the dielectric permittivity of a gold layer were calculated at $1064 \mathrm{~nm}$, and for thicknesses $t$ in the range 10-60 nm [Fig. 1(b)]. The real part $\operatorname{Re}\left[\varepsilon_{\mathrm{Au}}\right]$ is negative and changes insignificantly by only $\sim 0.9 \%$. This is because the frequency of electrons collisions $\gamma_{\mathrm{f}}$ is much less than telecom optical frequencies:

$\gamma_{\mathrm{f}} \sim 17.2 \times 10^{13} \mathrm{~s}^{-1}$ at $t=10 \mathrm{~nm}$ and decreases to $\sim 5.6 \times 10^{13} \mathrm{~s}^{-1}$ at $t=$ $60 \mathrm{~nm}$. In contrary, imaginary part $\operatorname{Im}\left[\varepsilon_{\mathrm{Au}}\right]$ changes by an order of magnitude. The obtained values for the linear refractive indices and dielectric permittivities of the constituent materials will be used further for calculation of the plasmonic waveguide dispersion.

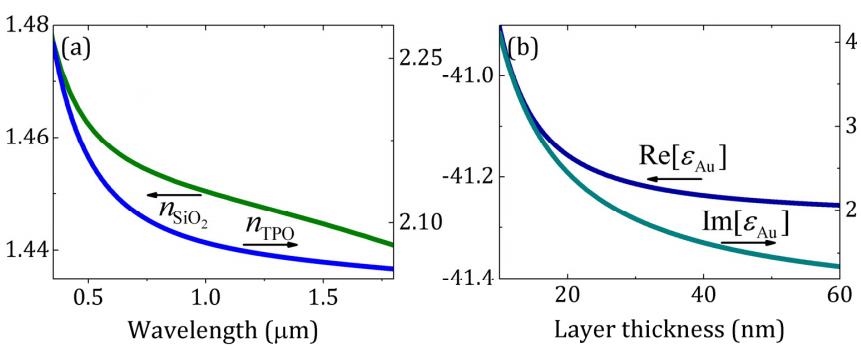

Fig. 1. (a) Linear refractive indices for $\mathrm{SiO}_{2}$ and $\mathrm{Ta}_{2} \mathrm{O}_{5}$. (b) Real and imaginary parts of the dielectric permittivity for a gold layer at 1064 $\mathrm{nm}$.

\section{STRIP PLASMONIC WAVEGUIDES}

The designed geometry of strip plasmonic waveguides (Fig. 2) contains the gold core with thickness $t$, tantalum pentoxide layers with thickness $t_{1}$, and thick silica claddings. The waveguide has the microscale width $w$ and millimeter scale length $l$. The thickness of the $\mathrm{SiO}_{2}$ claddings is large enough to cover the LRSPP mode distribution in the $y z$-plane. The propagation constant $\beta$ of the plasmonic mode can be calculated by the effective index method [24]. First, the propagation constant $\beta_{\infty}$ of the LRSPP mode is derived, assuming an infinitely wide $(w=\infty)$ slab waveguide.

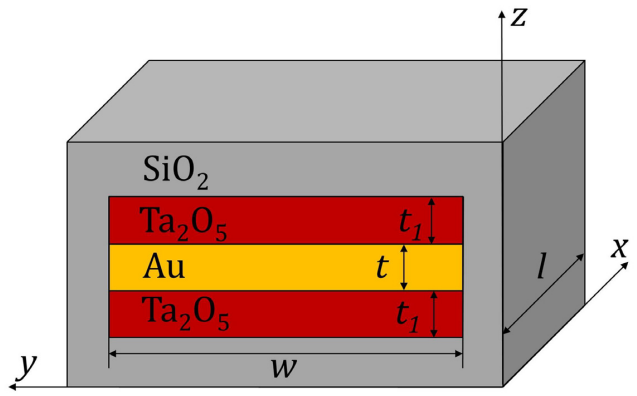

Fig. 2. Geometry of the strip plasmonic waveguide.

By using Maxwell's equations and boundary conditions it is straightforward to show that the dispersion equation for propagation constant $\beta_{\infty}$ in the symmetric five-layer system is given by:

$$
\frac{k_{3}}{\varepsilon_{3}} \tanh \left(\frac{t}{2} k_{3}\right)=-\frac{k_{2}}{\varepsilon_{2}} \frac{\left(\frac{k_{2}}{\varepsilon_{2}}+\frac{k_{1}}{\varepsilon_{1}}\right) e^{k_{2} t_{1}}-\left(\frac{k_{2}}{\varepsilon_{2}}-\frac{k_{1}}{\varepsilon_{1}}\right) e^{-k_{2} t_{1}}}{\left.\left(\frac{k_{2}}{\varepsilon_{2}}\right) \frac{k_{1}}{\varepsilon_{1}}\right) e^{k_{2} t_{1}}+\left(\frac{k_{2}}{\varepsilon_{2}}-\frac{k_{1}}{\varepsilon_{1}}\right) e^{-k_{2} t_{1}}}
$$

Here $k_{j}=\sqrt{\beta_{\infty}^{2}-\varepsilon_{j} \omega^{2} / c^{2}}(j=1,2,3)$ are the wave vectors for silica, tantalum pentoxide and gold, respectively. The dispersion equation is solved numerically for $\beta_{\infty}$, and the effective index of the LRSPP mode is $n_{\text {eff }}=\beta_{\infty} c / \omega$. Then, effective index $n_{\text {eff }}$ of the slab waveguide is used to determine the propagation constant $\beta$ of the plasmonic mode in the finite-width strip waveguide by solving the standard dispersion equation [24]:

$$
\sqrt{\frac{\omega^{2} n_{\mathrm{eff}}{ }^{2}}{c^{2}}-\beta^{2}} \tan \left(\frac{w}{2} \sqrt{\frac{\omega^{2} n_{\mathrm{eff}}{ }^{2}}{c^{2}}-\beta^{2}}\right)=\sqrt{\beta^{2}-\frac{\omega^{2} n_{\mathrm{SiO}_{2}}{ }^{2}}{c^{2}}} .
$$

The normalized propagation constant $\bar{\beta}=\beta c / \omega=n_{\text {eff } 1}$ corresponds to the effective refractive index of the LRSPP mode. Equation (8) has several roots, which correspond to different LRSPP spatial modes. The fundamental mode has the largest value of the effective refractive index $n_{\text {eff }}$ [25], and the effective refractive indices of other roots are in the range $n_{1}<n_{\text {eff } 1}<n_{\text {eff }}$. We will focus further on the fundamental mode, which is a transverse magnetic (TM) mode with the magnetic field pointed along the $y$-direction, and the electric field pointed along the $z$ - and $x$-directions. The real part of the effective refractive index $\operatorname{Re}\left[n_{\text {eff1 }}\right]$ and imaginary part of the propagation constant $\operatorname{Im}[\beta]$ were calculated in the wavelength range 800-1550 nm, and for thicknesses $t=22,27$, and $35 \mathrm{~nm}$ (Fig. 3). These specific values of the thickness were selected mainly to follow the recent experimental study [4]. They are above the clustering threshold and small enough to provide low-loss propagation of the LRSPP mode. The imaginary part of the propagation constant defines the linear absorption coefficient $\alpha=2 \operatorname{Im}[\beta]$ of the plasmonic mode [22]. As expected, the thinner gold layers have lower attenuation, and the LRSPP mode has a larger propagation distance, respectively. The $\mathrm{Ta}_{2} \mathrm{O}_{5}$ thickness $t_{1}=26 \mathrm{~nm}$ and the strip width $w=10 \mu \mathrm{m}$ were used in the calculations.
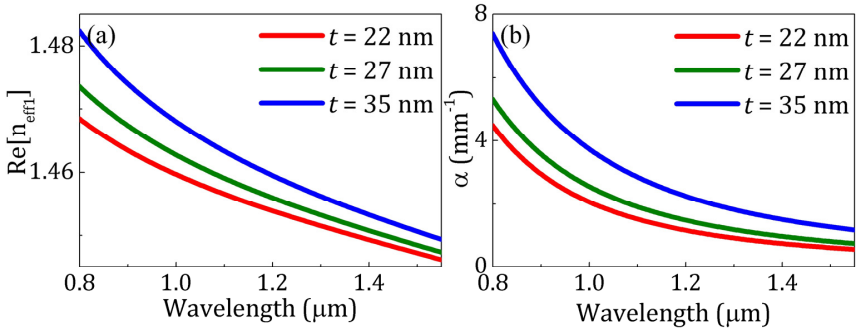

Fig. 3. (a) Effective index and (b) linear absorption coefficient vs. wavelength.

Further we focus on the dominating electric field component $E_{\mathrm{z}}$.

The normalized electric field intensity distribution $\left|E_{z}(y, z)\right|^{2}$ was calculated for the LRSPP mode in the transverse sections of the waveguide (Fig. 4). In the $x y$-plane, the distribution function experiences a step-like behavior at the interfaces, both $\mathrm{Au} \backslash \mathrm{Ta}_{2} \mathrm{O}_{5}$, and $\mathrm{Ta}_{2} \mathrm{O}_{5} \backslash \mathrm{SiO}_{2}$ [Fig. 4(a), Fig. 4(c)]. Thickness $t$ does not affect the intensity distribution in the $z$-plane [Fig. 4(b)]. The two-dimensional 
function $E_{\mathrm{z}}(y, z)$ with a good accuracy can be expressed in terms of a product of the one-dimensional distribution functions: $E_{\mathrm{z}}(y, z)=E_{\mathrm{z}}(y) E_{\mathrm{z}}(z)$. In fact, the value of $\beta_{\infty}$ for the infinitely wide slab waveguide differs by less than one percent from the value of $\beta$ for a finite-width strip waveguide, thus justifying the variables $y$ and $z$ separation in the expression for the two-dimensional distribution function. This property of the distribution function will be used in the further calculation of the field integrals for the constituent materials: gold, tantalum pentoxide and silica.

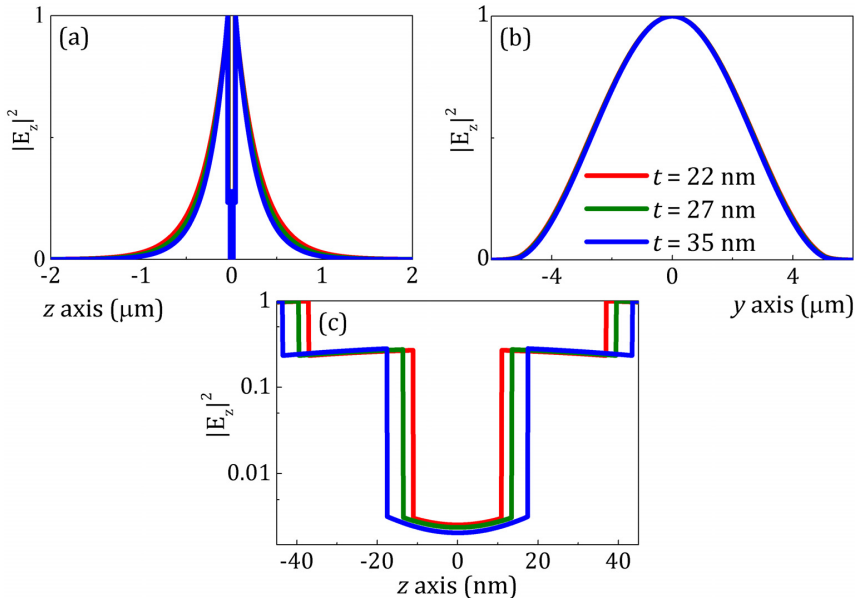

Fig. 4. Intensity distributions in (a) $z$ axis, (b) $y$ axis, and (c) zoom on the interfaces.

\section{CHROMATIC DISPERSION}

The first and second derivatives of the real part of the propagation constant define the lowest-order chromatic dispersion in plasmonic waveguides. The first derivative $\beta_{1}=d \beta / d \omega$ is related to group velocity $v_{\mathrm{g}}$ and group index $n_{\mathrm{g}}$ of the LRSPP mode as $\beta_{1}=1 / v_{\mathrm{g}}=n_{\mathrm{g}} / c$ [3]. The second derivative $\beta_{2}=d^{2} \beta / d \omega^{2}$ (also referred as the group velocity dispersion (GVD) parameter) is related to the characteristic dispersion length $L_{\mathrm{D}}$ of the plasmonic mode as $L_{\mathrm{D}}=T_{0}^{2} /\left|\beta_{2}\right|$, where $T_{0}$ is the $1 / \mathrm{e}$-intensity value of a Gaussian input optical pulse. Group index $n_{\mathrm{g}}$ and GVD parameter $\beta_{2}$ were calculated in the range $800-2000 \mathrm{~nm}$ (Fig. 5). The average value of $v_{\mathrm{g}}$ at $1064 \mathrm{~nm}$ is $\sim 67 \%$ of the vacuum speed of light. The values of the zero dispersion wavelength of the plasmonic mode are $\sim 1.7 \mu \mathrm{m}$, $\sim 1.8 \mu \mathrm{m}$, and $\sim 1.93 \mu \mathrm{m}$ for the gold layer of thicknesses 22,27 , and 35 $\mathrm{nm}$, respectively. The zero dispersion wavelength at $\sim 1.55 \mu \mathrm{m}$ can be achieved in plasmonic waveguides with the gold layer thickness 15 $\mathrm{nm}$. The magnitude of $\beta_{2}$ is ten times larger $\left(\sim 10^{-4} \mathrm{ps}^{2} / \mathrm{mm}=10^{2}\right.$ $\left.\mathrm{ps}^{2} / \mathrm{km}\right)$ than typical values for single-mode optical fibers $(\sim 10$ $\mathrm{ps}^{2} / \mathrm{km}$ ) [3]. Dispersion length $L_{\mathrm{D}}$ for 3 ps pulses is much larger ( 200-1000 $\mathrm{mm})$ than physical length of the waveguide $l(\sim 1 \mathrm{~mm})$. This means that the chromatic dispersion has a small effect on the propagation of picosecond optical pulses in the plasmonic waveguide.

The situation is different in the case of femtosecond optical pulses, when the dispersion length is comparable with the waveguide length. The chromatic dispersion can significantly affect the propagation of femtosecond optical pulses in a plasmonic waveguide, and many interesting phenomena can arise. In this paper we will focus on picosecond optical pulses, when the chromatic dispersion effect has a minor role. The case of femtosecond optical pulses will be discussed elsewhere.
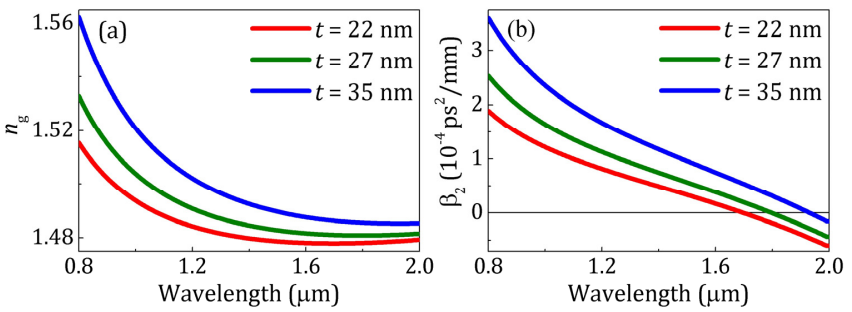

Fig. 5. (a) Group index and (b) GVD parameter vs. wavelength.

\section{NONLINEAR PARAMETERS}

For the quantitative analysis of effective nonlinear properties of the LRSPP mode in the waveguide we employed the field integrals in each material section given by the following expressions:

$$
\begin{gathered}
\theta_{\mathrm{Au}}=\frac{\int_{-t / 2}^{t / 2}\left|E_{z}\right|^{2} d z}{\int_{-\infty}^{+\infty}\left|E_{\mathrm{z}}\right|^{2} d z}, \\
\theta_{\mathrm{Ta}_{2} \mathrm{O}_{5}}=\frac{2 \int_{t / 2}^{t / 2+t_{1}}\left|E_{\mathrm{z}}\right|^{2} d z}{\int_{\mathrm{SiO}_{2}}^{+\infty}\left|E_{\mathrm{z}}\right|^{2} d z}, \\
\int_{t / 2+t_{1}}^{+\infty}\left|E_{\mathrm{z}}\right|^{2} d z \\
\int_{-\infty}^{+\infty}\left|E_{\mathrm{z}}\right|^{2} d z
\end{gathered}
$$

The initial two-dimensional integrals over the plasmonic mode area in the $y z$-plane were reduced to one-dimensional integrals over the $z$ coordinate by applying the variables separation justified above: $E_{\mathrm{z}}(y, z)=E_{\mathrm{z}}(y) E_{\mathrm{z}}(z)$. In fact, the one-dimensional integrals over the $y$-coordinate have the same values in the numerator and denominator and are canceled due to the waveguide symmetry.

The real and imaginary parts of effective third-order susceptibility $\chi^{(3)}$ of the LRSPP mode are expressed as follows:

$$
\begin{gathered}
\operatorname{Re}\left[\chi^{(3)}\right]= \\
\theta_{\mathrm{Au}} \operatorname{Re}\left[\chi_{3}^{(3)}\right]+\theta_{\mathrm{Ta}_{2} \mathrm{O}_{5}} \chi_{2}^{(3)}+\theta_{\mathrm{SiO}_{2}} \chi_{1}^{(3)}, \\
\operatorname{Im}\left[\chi^{(3)}\right]=\theta_{\mathrm{Au}} \operatorname{Im}\left[\chi_{3}^{(3)}\right],
\end{gathered}
$$

where $\chi_{1}^{(3)}$ is the third-order susceptibility for the $\mathrm{SiO}_{2}$ claddings [26], $\chi_{2}^{(3)}$ is the third-order susceptibility for the $\mathrm{Ta}_{2} \mathrm{O}_{5}$ layers [27] and 
$\chi_{3}^{(3)}$ is the third-order susceptibility for the Au layer. The third-order susceptibility for bulk gold is given by the following expression [15]:

$$
\chi_{\infty}^{(3)}=\frac{1}{2} \varepsilon_{0} \omega \operatorname{Im}\left[\varepsilon_{\mathrm{Au}}\right] \tau_{\mathrm{r}} \tau_{\mathrm{th}}\left(\frac{\gamma_{\mathrm{e}}}{C_{\mathrm{e}}}-\frac{\gamma_{\text {lat }}}{C_{\text {lat }}}\right) \frac{\partial \varepsilon_{\mathrm{inter}}}{\partial T_{\mathrm{e}}},
$$

where $C_{\mathrm{e}}$ and $C_{\text {lat }}$ are the electronic and lattice heat capacities per unit volume of gold, $\gamma_{\mathrm{e}}$ and $\gamma_{\text {lat }}$ are the electron and lattice thermalization rates, $\tau_{\text {th }}=1 /\left(\gamma_{\mathrm{e}}+\gamma_{\text {lat }}\right)$ is the decay time for nonthermalized electrons, $\tau_{\mathrm{r}}=C_{\mathrm{e}} C_{\text {lat }} /\left[C\left(C_{\mathrm{e}}+C_{\text {lat }}\right)\right]$ is the decay time for thermalized electrons, $C$ is the electron-phonon coupling coefficient, and $\partial \varepsilon_{\text {inter }} / \partial T_{\mathrm{e}}$ is the complex value of interband thermomodulational derivative. To calculate the thickness dependent thirdorder susceptibility for the gold layer $\chi_{3}^{(3)}$, one can substitute the thickness dependent permittivity $\varepsilon_{\text {Au }}$ from Eq. (6) into Eq. (15) and obtain the following expression:

$$
\chi_{3}^{(3)}=\chi_{\infty}^{(3)}\left(1+\frac{v_{\mathrm{F}}}{\gamma_{\infty} t}\right) .
$$

In the derivation of Eq. (15) we assumed all parameters in the twotemperature model, except $\operatorname{Im}\left[\varepsilon_{\mathrm{Au}}\right]$, were being thickness independent. For very small thicknesses, the model must be revised by using the nonlocal and quantum approaches $[6,13]$. The calculated real and imaginary parts of $\chi_{3}^{(3)}$ for layer thickness $t$ in the range 10$60 \mathrm{~nm}$ (Fig. 6) indicate the increment of the third-order susceptibility in $\sim 3$ times as the layer thickness decreases from $60 \mathrm{~nm}$ to $10 \mathrm{~nm}$. The horizontal asymptotes in Fig. 6 correspond to the value of third-order susceptibility $\chi_{\infty}^{(3)}$ for bulk gold at $1064 \mathrm{~nm}$ [15]. The real part is $\operatorname{Re}\left[\chi_{\infty}^{(3)}\right]=4.6 \times 10^{-17} \mathrm{~m}^{2} / \mathrm{V}^{2}$, and the imaginary part is $\operatorname{Im}\left[\chi_{3}^{(3)}\right]=$ $4.8 \times 10^{-18} \mathrm{~m}^{2} / \mathrm{V}^{2}$. The real part $\operatorname{Re}\left[\chi_{\infty}^{(3)}\right]$ is $\sim 10$ times larger in magnitude than the imaginary part $\operatorname{Im}\left[\chi_{3}^{(3)}\right]$ for the same values of the layer thickness. Figure 6 also shows the thickness limitations. The Drude model (Eq. (3)-(5)) may be inapplicable for the layer thicknesses below 10-15 nm due to the possible quantum effects [6], nonlocal effects [13], and layer clustering [20]. Conversely, the coupled LRSPP mode collapses at the layer thicknesses above $50-60 \mathrm{~nm}$ due to the small penetration depth of the surface electromagnetic waves in metal [22]. Therefore, the effective range of $t$ is $\sim 15-50 \mathrm{~nm}$ for experimental studies.

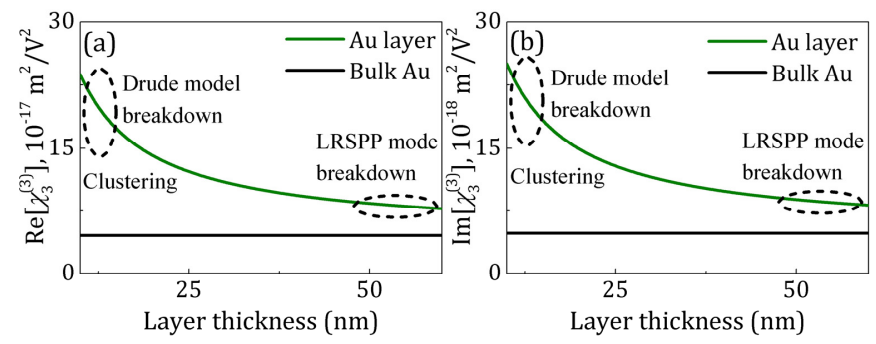

Fig. 6. (a) Real part and (b) imaginary part of the third-order susceptibility for Au layers at $1064 \mathrm{~nm}$.
The literature values of $\chi_{\infty}^{(3)}$ at $1550 \mathrm{~nm}$ are mostly the same as at $1064 \mathrm{~nm}$ [15], but the linear absorption in the waveguide is somewhat lower [Fig. 3(b)]. The wavelength $1550 \mathrm{~nm}$ can be a good option for an experimental study of nonlinear effects in the gold strip plasmonic waveguides. Moreover, for this wavelength anomalous waveguide GVD can be found, making it possible to excite solitons. For the wavelength $800 \mathrm{~nm}$, although the value of $\operatorname{Re}\left[\chi_{\infty}^{(3)}\right]$ is $\sim 40 \%$ larger than at $1064 \mathrm{~nm}$, the value of $\operatorname{Im}\left[\chi_{\infty}^{(3)}\right]$ is $\sim 5$ times larger. This means that nonlinear absorption will dominate the nonlinear dynamics.

Effective nonlinear refractive index $n_{\text {2eff }}$, nonlinear absorption coefficient $\beta$, and nonlinear parameter $\gamma$ of the plasmonic mode are expressed by the following expressions [28]:

$$
\begin{gathered}
n_{2 \mathrm{eff}}=\frac{3}{4 \varepsilon_{0} c n_{0}^{2}} \operatorname{Re}\left[\chi^{(3)}\right], \\
\beta=\frac{\beta_{\mathrm{c}}}{A_{\mathrm{eff}}}=\frac{3 \omega}{2 \varepsilon_{0} c^{2} n_{0}^{2} A_{\mathrm{eff}}} \operatorname{Im}\left[\chi^{(3)}\right], \\
\gamma=\frac{\omega}{c} \frac{n_{2 \mathrm{eff}}}{A_{\mathrm{eff}}}
\end{gathered}
$$

where $\beta_{\mathrm{c}}$ is the conventional definition of the nonlinear absorption coefficient [28], and $A_{\text {eff }}$ is the effective area of the plasmonic mode calculated by the following formula [3]:

$$
A_{\mathrm{eff}}=\frac{\left(\int_{-\infty}^{+\infty} \int_{-\infty}^{+\infty}\left|E_{\mathrm{z}}(y, z)\right|^{2} d y d z\right)^{2}}{\int_{-\infty}^{+\infty} \int_{-\infty}^{+\infty}\left|E_{\mathrm{z}}(y, z)\right|^{4} d y d z} .
$$

The calculated values of the field integrals $\theta_{\mathrm{Au}}, \theta_{\mathrm{Ta}_{2} \mathrm{O}_{5}}, \theta_{\mathrm{SiO}_{2}}$, effective linear refractive index $n_{\text {effl }}$ and third-order susceptibility $\chi_{3}^{(3)}$ of the LRSPP mode, and linear refractive index $n_{\mathrm{Au}}$ of the gold layer for layer thicknesses $t=22,27$ and $35 \mathrm{~nm}$ are summed up in Table 1. The variation of each parameter was calculated as a function of the thickness variation $\delta_{\mathrm{t}}=1 \mathrm{~nm}$. In all the calculations, the same values of the $\mathrm{Ta}_{2} \mathrm{O}_{5}$ layers thickness $t_{1}=26 \mathrm{~nm}$, waveguide strip width $w=10 \mu \mathrm{m}$, and wavelength $\lambda=1064 \mathrm{~nm}$ were used. The literature values of the dielectrics third-order susceptibilities are $\chi_{1}^{(3)}=2.07 \times$ $10^{-22} \mathrm{~m}^{2} / \mathrm{V}^{2}$ for $\mathrm{SiO}_{2}$, and $\chi_{2}^{(3)}=1.12 \times 10^{-20}$ for $\mathrm{Ta}_{2} \mathrm{O}_{5}$. The field integrals values indicate that the most of the electric field of the LRSPP mode (around 97\%) is situated in the $\mathrm{SiO}_{2}$ claddings. However, the contribution terms from tantalum pentoxide $\theta_{\mathrm{Ta}_{2} \mathrm{O}_{5}} \chi_{2}^{(3)}$ and silica $\theta_{\mathrm{SiO}_{2}} \chi_{1}^{(3)}$ to the real part of the third-order susceptibility $\operatorname{Re}\left[\chi^{(3)}\right]$ are of the same order of magnitude $\left(\sim 3 \times 10^{-22} \mathrm{~m}^{2} / \mathrm{V}^{2}\right.$ and $\sim 2 \times 10^{-22}$ $\mathrm{m}^{2} / \mathrm{V}^{2}$, respectively). The contribution from the gold layer is $\sim$ two 
orders of magnitude larger $\left(\sim 2 \times 10^{-20} \mathrm{~m}^{2} / \mathrm{V}^{2}\right)$, and is therefore dominating. The imaginary part of the third-order susceptibility $\operatorname{Im}\left[\chi^{(3)}\right]$ is solely determined by the imaginary part of the thirdorder susceptibility $\operatorname{Im}\left[\chi_{3}^{(3)}\right]$ of the gold layer as the chosen wavelength has a photon energy that is well below half of the optical bandgap of silica and tantalum pentoxide, nullifying their respective imaginary nonlinear susceptibility. Thus, the dominant role of the nonlinearity of the gold layer in the effective nonlinearity of the plasmonic mode is confirmed. This is in accordance with the recent theoretical results published by Baron et al. for other plasmonic systems [29].

Table 1. Parameters of the waveguide: $\theta_{\mathrm{Au}}, \theta_{\mathrm{Ta}_{2} \mathrm{O}_{5}}, \theta_{\mathrm{SiO}_{2}}, n_{\text {eff1 }}$, $n_{\mathrm{Au}}, A_{\mathrm{eff}}, \beta, \gamma, \chi_{3}^{(3)}$ for different gold layer thicknesses.

\begin{tabular}{|c|c|c|c|}
\hline$t, \mathrm{~nm}$ & $35 \pm 1$ & $27 \pm 1$ & $22 \pm 1$ \\
\hline$\theta_{\mathrm{Au}}, 10^{-3}$ & $0.1872 \pm 0.0055$ & $0.1397 \pm 0.0062$ & $0.1084 \pm 0.0063$ \\
\hline$\theta_{\mathrm{Ta}_{2} \mathrm{O}_{5}}$ & $0.0298 \pm 0.0004$ & $0.0263 \pm 0.0004$ & $0.0239 \pm 0.0005$ \\
\hline$\theta_{\mathrm{SiO}_{2}}$ & $0.9701 \pm 0.0005$ & $0.9736 \pm 0.0005$ & $0.9760 \pm 0.0005$ \\
\hline $\operatorname{Re}\left[n_{\mathrm{effl}}\right]$ & $1.4649 \pm 0.0003$ & $1.4603 \pm 0.0003$ & $1.4576 \pm 0.0003$ \\
\hline $\mathrm{Im}\left[n_{\mathrm{effl}}\right], 10^{-2}$ & $0.0265 \pm 0.0009$ & $0.0179 \pm 0.0009$ & $0.0143 \pm 0.0009$ \\
\hline $\operatorname{Re}\left[n_{\mathrm{Au}}\right]$ & $0.1352 \pm 0.0020$ & $0.1570 \pm 0.0034$ & $0.1786 \pm 0.0050$ \\
\hline $\mathrm{Im}\left[n_{\mathrm{Au}}\right]$ & $6.4223 \pm 0.0001$ & $6.4208 \pm 0.0003$ & $6.4192 \pm 0.0004$ \\
\hline$A_{\mathrm{eff}}, \mu \mathrm{m}^{2}$ & $9.17 \pm 0.11$ & $10.24 \pm 0.16$ & $11.15 \pm 0.20$ \\
\hline$\beta, 1 /(\mathrm{W} \cdot \mathrm{m})$ & $0.139 \pm 0.005$ & $0.095 \pm 0.005$ & $0.069 \pm 0.005$ \\
\hline$\gamma, 1 /(\mathrm{W} \cdot \mathrm{m})$ & $0.77 \pm 0.03$ & $0.52 \pm 0.03$ & $0.38 \pm 0.03$ \\
\hline $\operatorname{Re}\left[\chi_{3}^{(3)}\right], \mathrm{m}^{2} / \mathrm{V}^{2}$ & $10.03 \times 10^{-17}$ & $11.65 \times 10^{-17}$ & $13.27 \times 10^{-17}$ \\
\hline $\operatorname{Im}\left[\chi_{3}^{(3)}\right], \mathrm{m}^{2} / \mathrm{V}^{2}$ & $10.58 \times 10^{-18}$ & $12.29 \times 10^{-18}$ & $14.00 \times 10^{-18}$ \\
\hline
\end{tabular}

\section{PULSE PROPAGATION EQUATION}

Once the mode properties of the strip plasmonic waveguide are known, the propagation dynamics of these modes is well described by the nonlinear Schrödinger equation [3]:

$$
\frac{\partial A}{\partial x}=i \gamma|A|^{2} A-\frac{\alpha}{2} A-\frac{\beta}{2}|A|^{2} A,
$$

where $A(x, T)$ is the complex amplitude of an optical pulse, $x$ is the propagation coordinate, $T$ is the time coordinate in the co-moving frame of the input pulse. The first and third term correspond to the self-phase modulation (SPM) and nonlinear absorption, respectively. The chromatic dispersion has been neglected, which is a good approximation as dispersion length $L_{\mathrm{D}}$ for picosecond optical pulses in a plasmonic waveguide is much larger than its physical length $l$. The formal solution to Eq. (20) is $A(x, T)=\sqrt{P(x, T)} \exp \left[i \phi_{\mathrm{NL}}(x, T)\right]$, where $P(x, T)$ is the pulse power amplitude and $\phi_{\mathrm{NL}}(x, T)$ is the pulse nonlinear phase. Although this has been studied extensively in the literature, we are here dealing with the particular case where dispersion is negligible and where linear and nonlinear absorption is significant, which makes it worth to revise the results. Substituting this solution in Eq. (20) gives the following system of differential equations:

$$
\left\{\begin{array}{c}
\frac{\partial P(x, T)}{\partial x}=-\alpha P(x, T)-\beta|P(x, T)|^{2} \\
\frac{\partial \phi_{\mathrm{NL}}(x, T)}{\partial x}=\gamma P(x, T) .
\end{array}\right.
$$

The system is straightforward to solve for the Gaussian pulse initial conditions: $P(0, T)=P_{0} \exp \left(-T^{2} / T_{0}^{2}\right)$ and $\phi_{\mathrm{NL}}(0, T)=0$ :

$$
\left\{\begin{array}{c}
P(x, T)=\frac{P_{0} e^{-T^{2} / T_{0}^{2}} e^{-\alpha x}}{1+\beta P_{0} e^{-T^{2} / T_{0}^{2}}\left(1-e^{-\alpha x}\right) / \alpha}, \\
\phi_{\mathrm{NL}}(x, T)=\frac{\gamma}{\beta} \ln \left[1+\beta P_{0} e^{-T^{2} / T_{0}^{2}}\left(1-e^{-\alpha x}\right) / \alpha\right] .
\end{array}\right.
$$

These solutions describe how peak power $P(x, T)$ and phase $\phi_{\mathrm{NL}}(x, T)$ of optical pulses change in the time domain upon propagation. For practical purposes, the expression for $P(x, T)$ can be modified to obtain a formula for the average power of light at the output of the plasmonic waveguide, which is the observable and measurable quantity. The equation for the pulse energy $E(x)$ is expressed as:

$$
\begin{gathered}
E(x)=\int_{-\infty}^{+\infty}|A(x, T)|^{2} d T \\
=\int_{-\infty}^{+\infty} \frac{P_{0} T_{0} e^{-\alpha x} e^{-T^{2} / T_{0}^{2}}}{1+\beta P_{0} e^{-T^{2} / T_{0}^{2}}\left(1-e^{-\alpha x}\right) / \alpha} d\left(T / T_{0}\right) .
\end{gathered}
$$

It is straightforward to show that if the integral in Eq. (23) is expanded in the Taylor series up to the second order term, this equation can be simplified to the following form:

$$
E(x)=P_{0} T_{0} e^{-\alpha x}\left(\sqrt{\pi}-\frac{\sqrt{\pi} \beta P_{0}}{\sqrt{2}} \frac{1-e^{-\alpha x}}{\alpha}\right),
$$

where $E(x)=\bar{P}(x) / f_{\text {rep }}$ is the pulse energy in terms of average power $\bar{P}(x)$ and laser repetition rate $f_{\text {rep }}$. Peak power $P_{0}$ is related to average input power $\overline{P_{0}}$ as $P_{0}=\overline{P_{0}} /\left(\sqrt{\pi} T_{0} f_{\text {rep }}\right)$. By substituting the expression for the pulse energy and peak power in Eq. (24), and accounting that $\exp (-\alpha x)<<1$ at the waveguide output $(x=l)$ due to the large linear attenuation [Fig. 3(b)], the following equation for average output power $\bar{P}(l)$ is obtained:

$$
\bar{P}(l)=\overline{P_{0}} e^{-\alpha l}-\frac{\sqrt{\pi} \beta{\overline{P_{0}}}^{2}}{\sqrt{2} \alpha T_{0} f_{\text {rep }}} e^{-\alpha l} .
$$


This equation can be used for practical applications since the average input and output power are the directly measurable quantities. It shows that average output power $\bar{P}(l)$ from the plasmonic waveguide of length $l$ depends nonlinearly on the average input power $\overline{P_{0}}$, and an asymptotic saturation of function $\bar{P}(l)\left\{\overline{P_{0}}\right\}$ is expected at high values of $\overline{P_{0}}$. The nonlinear trend $\bar{P}(l)\left\{\overline{P_{0}}\right\}$ depends on linear absorption coefficient $\alpha$, laser repetition rate $f_{\text {rep }}$, pulse duration $T_{0}$, nonlinear parameter $\beta$, and length $l$ of the waveguide. The calculated power trends for different thicknesses $t=$ 22, 27 and $35 \mathrm{~nm}$ (Fig. 7) confirm the expected nonlinear behavior. The nonlinear term in Eq. (25) becomes important for values of $\overline{P_{0}}$ above 0.15-0.2 W. The possible saturation plateau is achievable at values of $\overline{P_{0}}$ above $0.5 \mathrm{~W}$. The obtained nonlinear functions $\bar{P}(l)\left\{\overline{P_{0}}\right\}$ clearly depend on gold thickness $t$, the saturation is achieved faster for thinner waveguides. It confirms that gold layer thickness $t$ is a crucial parameter for $\chi^{(3)}$ of the LRSPP mode. The following parameters were used in the calculations: waveguide width $w=10 \mu \mathrm{m}$ and length $l=3 \mathrm{~mm}, \mathrm{Ta}_{2} \mathrm{O}_{5}$ thickness $t_{1}=26 \mathrm{~nm}$, laser peak wavelength $\lambda=$ $1064 \mathrm{~nm}$, repetition rate $f_{\text {rep }}=78 \mathrm{MHz}$, and FWHM pulse duration $\Delta T=2 \sqrt{\ln (2)} T_{0} \approx 3$ ps.

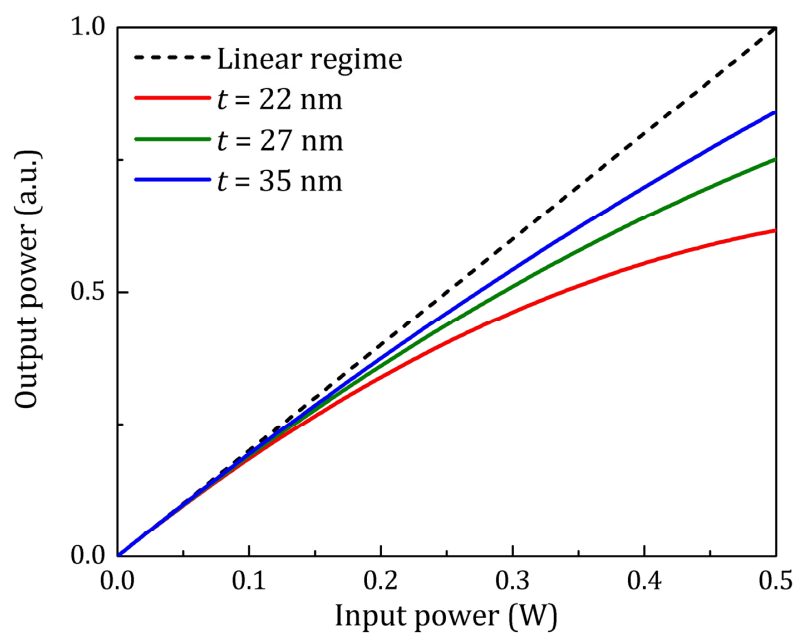

Fig. 7. Nonlinear power trends for different gold layer thicknesses at $1064 \mathrm{~nm}$. Black line corresponds to the linear LRSPP regime.

The second nonlinear effect concerns the LRSPP mode spectral features. The pulse power spectral density (PSD) in $\mathrm{W} / \mathrm{Hz}$ is given by the squared Fourier transform of pulse amplitude $A(x, T)$ as follows:

$$
\operatorname{PSD}(x, \omega)=f_{\text {rep }}\left|\int_{-\infty}^{+\infty} A(x, T) e^{i \omega T} d T\right|^{2}
$$

By substituting the expressions for the pulse power amplitude and phase in Eq. (26), the final formula for the PSD is obtained:

$$
\operatorname{PSD}(l, \omega)=f_{\text {rep }}\left|\int_{-\infty}^{+\infty}\left(\frac{P_{0} e^{-\frac{T^{2}}{T_{0}^{2}}} e^{-\alpha l}}{1+\beta P_{0} e^{-\frac{T^{2}}{T_{0}^{2}}} L_{\text {eff }}}\right)^{1 / 2} e^{i \frac{\gamma}{\beta} \ln \left[1+L_{\mathrm{eff}} \beta P_{0} e^{\frac{T^{2}}{T_{0}^{2}}}\right]} e^{i\left(\omega-\omega_{\mathrm{c}}\right) T} d T\right|^{2},
$$

where $\omega_{\mathrm{c}}=2 \pi c / \lambda$ is the carrier frequency, which corresponds to the peak wavelength $1064 \mathrm{~nm}$, and $L_{\text {eff }}=\left(1-e^{-\alpha l}\right) / \alpha$ is the effective waveguide length. The numerical modeling based on Eq. (27) for the gold layer thickness $t=22 \mathrm{~nm}$ (Fig. 8) shows the nonlinear spectral broadening and splitting of an input Gaussian pulse, depending on input average power $\overline{P_{0}}$. The pulse splitting occurs at values of $\overline{P_{0}} \sim 0.2 \mathrm{~W}$. This nonlinear effect was observed in our recent experiment [4]. The further increase of $\overline{P_{0}}$ leads to the splitting and formation of more SPM peaks. The cases of other thicknesses $(27 \mathrm{~nm}$, $35 \mathrm{~nm}$ ) and wavelengths (800 nm, $1550 \mathrm{~nm})$ are qualitatively the same. The splitting occurs at values of $\overline{P_{0}} \sim 0.1 \mathrm{~W}$ at $1550 \mathrm{~nm}$, and $\overline{P_{0}}$ $\sim 1.2 \mathrm{~W}$ at $800 \mathrm{~nm}$.

The obtained spectral features are in accordance with the theoretical results for nonlinear properties of silicon waveguides [30, 31]. The principal difference here is that a plasmonic waveguide has much higher linear absorption and shorter physical length than an ordinary silicon waveguide. However, the comparable nonlinear spectral broadening can be achieved. In the case of optical fibers, the required waveguide length can reach hundreds of meters or more to receive the desired nonlinear features. In this sense a plasmonic or silicon waveguide provides the same functionality, can be integrated on-chip and used for nonlinear purposes even with very short propagation lengths.

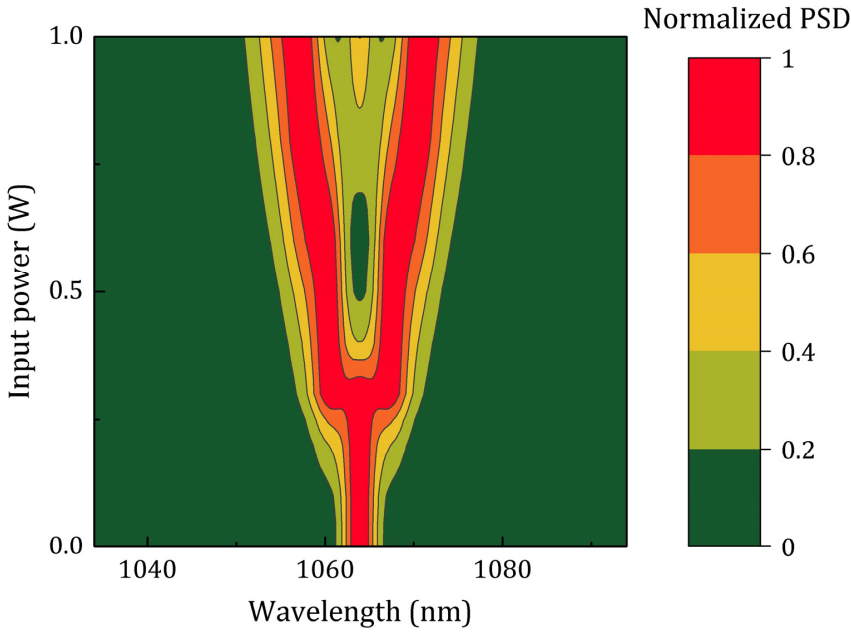

Fig. 8. SPM broadening of the input Gaussian pulse at $1064 \mathrm{~nm}$ using $t=22 \mathrm{~nm}$.

\section{CONCLUSIONS AND OUTLOOK}

In this paper we have presented the theoretical model of nonlinear behavior for plasmonic waveguides. The effective third-order susceptibility $\chi^{(3)}$ of the LRSPP mode is the crucial parameter for explanation of the nonlinear effects in plasmonic strip waveguides - 
nonlinear power absorption and nonlinear self-phase modulation of the LRSPP mode. The third-order susceptibility $\chi_{\mathrm{Au}}^{(3)}$ of the gold core has the dominant contribution to $\chi^{(3)}$ of the whole plasmonic mode. It was verified for three values of gold layer thicknesses $t=22,27$ and $35 \mathrm{~nm}$. The key point is that the nonlinear properties are thickness dependent and the third-order susceptibility of gold layers is inversely proportional to the thickness. Thin metal layers enhance the waveguide nonlinearity and provide effective nonlinear propagation of ultrafast optical pulses. The results confirmed the nonlinear power saturation that was observed in [4] for the average values of the input power $\overline{P_{0}}$ in the range above $0.15-0.2 \mathrm{~W}$, and for the high repetition rate $f_{\text {rep }}=78 \mathrm{MHz}$ picosecond laser pumping at $1064 \mathrm{~nm}$. In the same range an SPM effect was observed, leading to the LRSPP spectral broadening and splitting, again in accordance with our recent experimental results. The deep analogy with classical fiber-based nonlinear optics shows that plasmonic waveguides are potential candidates for nonlinear optics applications on a microscopic length scale.

One of the possible developments in this direction is the nonlinear analysis of higher order plasmonic modes. The current model is for the fundamental LRSPP mode, but other spatial modes can also be excited. These higher order modes will affect the dynamics by nonlinear cross-phase modulation [3]. Another development concerns the propagation of even shorter (femtosecond) pulses in the plasmonic waveguides. The chromatic dispersion effect may have a more dominant role than in the present case. The investigation of nonlinear propagation of femtosecond pulses in the plasmonic waveguides will be done elsewhere. The interaction of the nonlinear and GVD effects leads to many interesting optical phenomena in plasmonic nanostructures with their possible practical realization, for example, in optical communication systems. Our results indicate that the waveguide investigated here could have anomalous GVD at telecom Cband wavelengths, which means that solitons can be excited using femtosecond Er-fiber lasers. This has interesting nonlinear applications that promise well for plasmonic systems.

\section{References}

1. M. Kauranin and A. Zayats, "Nonlinear plasmonics," Nat. Photon. 6, 737748 (2012).

2. N. Rotenberg, M. Betz, and H. M. van Driel, "Nonlinear absorption in Au films: Role of thermal effects," Phys. Rev. Lett. 105, 017402 (2010).

3. G. P. Agrawal, Nonlinear Fiber Optics (Elsevier, 2013).

4. O. Lysenko, M. Bache, and A. Lavrinenko, "Third-order susceptibility of gold for ultrathin layers," Opt. Lett. 41, 2, 317-320 (2016).

5. A. Huck, D. Witthaut, S. Kumar, A. Soerensen, and U. L. Andersen, "Large optical nonlinearity of surface plasmon modes on thin gold films," Plasmonics 8, 4, 1597 (2013).

6. Z. Liu, Y. Xiao, and H. Qian, "Investigations of the optical properties of nanoscale gold films," in Advanced Photonics 2015, OSA Technical Digest (Optical Society of America, 2015), paper NM4C.1.

7. E. Xenogiannopoulou, P. Aloukos, S. Couris, E. Kaminska, A. Piotrowska, and E. Dynowska, "Third-order nonlinear optical properties of thin sputtered gold films," Opt. Commun. 275, 217 (2007).

8. A. Baron, T. B. Hoang, C. Fang, M. H. Mikkelsen, and D. R. Smith, "Ultrafast self-action of surface-plasmon polaritons at an air/metal interface," Phys. Rev. B 91, 195412 (2015).

9. K. F. MacDonald, Z. L. Samson, M. I. Stockman, and N. I. Zheludev, "Ultrafast active plasmonics," Nat. Photon. 3, 55-58 (2009).

10. P. Berini, "Long-range surface plasmon polaritons," Adv. Opt. Photon. 1, 3, 484-588 (2009).
11. D. Gramotnev and S. Bozhevolnyi, "Nanofocusing of electromagnetic radiation," Nat. Photon. 8, 13-22 (2014).

12. P. Berini, "Surface plasmon photodetectors and their applications," Laser Photon. Rev. 8, No. 2, 197-220 (2014).

13. S. Raza, S. Bozhevolnyi, M. Wubs, and N. A. Mortensen, "Nonlocal optical response in metallic nanostructures," J. Phys. Condens. Matter 27, 183204, 1-17 (2015).

14. P. Ginzburg, A. Krasavin, and A. Zayats, "Cascaded second-order surface plasmon solitons due to intrinsic metal nonlinearity," New J. Phys. 15, 013031 (2013).

15. A. Marini, M. Conforti, G. Della Valle, H. W. Lee, Tr. X. Tran, W. Chang, M. A. Schmidt, S. Longhi, P. St. J. Russell, and F. Biancalana, "Ultrafast nonlinear dynamics of surface plasmon polaritons in gold nanowires due to the intrinsic nonlinearity of metals," New J. Phys. 15, 013033 (2013).

16. M. Conforti and G. Della Valle, "Derivation of third-order nonlinear susceptibility of thin metal films as a delayed optical response," Phys. Rev. B 85, 245423 (2012).

17. I. H. Malitson, "Interspecimen comparison of the refractive index of fused silica," J. Opt. Soc. Am. 55, 10, 1205-1209 (1965).

18. T. J. Bright, J. I. Watjen, Z. M. Zhang, C. Muratore, A. A. Voevodin, D. I. Koukis, D. B. Tanner, and D. J. Arenas, "Infrared optical properties of amorphous and nanocrystalline $\mathrm{Ta}_{2} \mathrm{O}_{5}$ thin films," J. Appl. Phys. 114, 083515 (2013).

19. V. Klimov, Nanoplasmonics (Pan Stanford, 2014).

20. U. Kreibig and M. Vollmer, Optical Properties of Metal Clusters (Springer, 1995).

21. J. A. Gordon and R. W. Ziolkowski, "The design and simulated performance of a coated nano-particle laser," Opt. Express 15, 5, 26222653 (2007).

22. S. Maier, Plasmonics: Fundamentals and Applications (Springer, 2007).

23. R. Malureanu and A. Lavrinenko, "Ultra-thin films for plasmonics: a technology overview," Nanotechnol. Rev. 4, 3, 1-17 (2015).

24. P. Yeh, Optical Waves in Layered Media (Wiley, 1988).

25. R. G. Hunsperger, Integrated Optics: Theory and Technology (Springer, 2002).

26. D. Milam, "Review and assessment of measured values of the nonlinear refractive-index coefficient of fused silica," Appl. Opt. 37, 3, 546-550 (1998).

27. C. Tai, J. S. Wilkinson, N. M. B. Perney, M. C. Netti, F. Cattaneo, C. E. Finlayson, and J. J. Baumberg, "Determination of nonlinear refractive index in $\mathrm{Ta}_{2} \mathrm{O}_{5}$ rib waveguide using self-phase modulation," Opt. Express 12, 21, 5110-5116 (2004).

28. R. Boyd, Nonlinear Optics (Elsevier, 2008).

29. A. Baron, S. Larouche, D. J. Gauthier, and D. R. Smith, "Scaling of the nonlinear response of the surface plasmon polariton at a metal/dielectric interface," J. Opt. Soc. Am. B 32, 1, 9-14 (2015).

30. Q. Lin, O. J. Painter, and G. P. Agrawal, "Nonlinear optical phenomena in silicon waveguides: Modeling and applications," Opt. Express 15, 25, 16604-16644 (2007).

31. L. Yin and G. P. Agrawal, "Impact of two-photon absorption on self-phase modulation in silicon waveguides," Opt. Lett. 32, 14, $2031-2033$ (2007). 